\title{
POSOPERATORIO ENDOSCÓPICO TRANSNASAL POR POLIPOSIS: USO DE MONTELUKAST Y SEGUIMIENTO A LA RECURRENCIA
}

María Alejandra Taborda Samper MD*, Miguel Ángel González Romero MD**, Alejandro Motta Cortés MD***

\section{Resumen}

La poliposis nasal es uno de los motivos de consulta más frecuentes en rinosinusología con presentación insidiosa, alto impacto en la calidad de vida y poca respuesta a los tratamientos habituales. Objetivo: describir la recurrencia de poliposis nasal y el grado de control con montelukast en el posoperatorio de cirugía endoscópica transnasal en el Hospital de San José de Bogotá DC. Métodos: informe preliminar de estudio de tipo cohorte prospectiva con seguimiento a seis meses de doce pacientes llevados a cirugía por poliposis nasal, utilizando una dosis diaria de montelukast de $10 \mathrm{mg}$. Resultados: se obtuvo control sintomático satisfactorio con montelukast y esteroide tópico en el período posoperatorio en más de $\mathbf{7 5 \%}$ de los pacientes. Adecuada adherencia al tratamiento en más de $90 \%$ de los casos. Recurrencias tempranas (entre segundo y tercer meses) por nasofibrolaringoscopia en $33 \%$ de los doce pacientes tras seis meses de seguimiento. Conclusión: se reconoce el uso de montelukast como factor que sumado al tratamiento clásico con esteroide tópico nasal, logra un adecuado control sintomático y es promisorio en evitar la recurrencia.

Palabras clave: inhibidores de leucotrieno, montelukast, poliposis nasal.

Abreviaturas: NFL, nasofibrolaringoscopia.

\section{POST OPERATIVE PERIOD OF TRANSNASAL ENDOSCOPIC SURGERY FOR POLYPOSIS: USE OF MONTELUKAST AND RELAPSE FOLLOW UP}

\begin{abstract}
Nasal polyposis is one of the most common reasons for consultation in rhinosinusology with an insidious presentation with high impact on quality of life and poor response to common treatments. Objective: to describe relapse of nasal polyposis and level of control with the use of montelukast in the postoperative period of transnasal endoscopic surgery at Hospital de San José, Bogotá DC. Methods: preliminary report on a cohort prospective study with a 6-month followup of 12 patients operated for nasal polyposis, using a $10 \mathrm{mg}$ daily dose of montelukast. Results: a satisfactory symptomatic control was obtained with montelukast combined with topic steroids during the postoperative period in more than $75 \%$ of patients. Adherence to treatment was adequate in more than $90 \%$ of cases. Early relapses (between second and third month) were found by nasofibro-laryngoscopy in $33 \%$ of 12 patients after a 6-month follow-up. Conclusion: the use of montelukast is acknowledged as a factor which combined with the classical treatment with nasal topic steroids, achieves adequate symptomatic control and is promissory for relapse prevention.
\end{abstract}

Key words: leukotriene inhibitors, montelukast, nasal polyposis.

Fecha recibido: marzo 17 de 2010 - Fecha aceptado: mayo 12 de 2011

* Otorrinolaringóloga. Especialista en Cirugía plástica facial y maxilofacial. Hospital de San José. Instructora Asistente, Fundación Universitaria de Ciencias de la Salud, Bogotá DC. Colombia.
** Residente IV Otorrinolaringología. Fundación Universitaria de Ciencias de la Salud, Hospital de San José. Bogotá DC. Colombia.

*** Residente III Otorrinolaringología. Fundación Universitaria de Ciencias de la Salud, Hospital de San José. Bogotá DC. Colombia. 


\section{Introducción}

El término "pólipo" tiene origen griego. Polypus en latín significa muchos pies. Sorprende como esta connotación parece ser bien descriptiva en términos de la patogénesis de la poliposis nasal. De acuerdo con el documento "Ensayo de la posición europea sobre rinosinusitis y poliposis nasal" (EP3OS) emitido hace poco por la Academia Europea de Alergología e Inmunología Clínica y la Sociedad Europea de Rinología, la poliposis nasal se puede considerar como un subgrupo de la rinosinusitis crónica. Estudios endoscópicos e histológicos han evidenciado que corresponde a un proceso benigno con inflamación, acumulación de líquido extracelular y proliferación de mucosa y submucosa en la región turbinal y osteomeatal, siendo más frecuente en adultos que en niños. Por lo regular se presenta entre la tercera y la sexta décadas de la vida con una fuerte predominancia en hombres en proporción de 4:1.' La prevalencia está en el rango de $1 \%$ a $4,3 \%$.

La mayoría de los pólipos se originan en los pliegues del complejo osteomeatal y se extienden a la cavidad nasal causando obstrucción nasal, secreción, hiposmia, cefalea y reducción de la calidad de vida. Los pólipos nasales se caracterizan por edema tisular masivo, resultado de filtración de plasma a través de las uniones endoteliales de los vasos sanguíneos. La poliposis nasal es una enfermedad multifactorial que incluye anormalidades genéticas, inflamación crónica no infecciosa y asociación con procesos infecciosos. La mayoría de las teorías la considera como una manifestación final de inflamación crónica. Se asocian múltiples condiciones como rinitis alérgica y no alérgica, sinusitis alérgica fúngica, intolerancia al ASA, síndrome de Churg Strauss, fibrosis quística, disquinesia ciliar primaria y síndromes de Kartagener y de Young.

Con respecto a la clínica, se puede decir que esta no difiere mucho de los síntomas de la rinosinusitis crónica. Aunque puede ser asintomática, casi siempre se manifiesta por fatiga o cefalea global. Otras manifestaciones concomitantes son congestión nasal (progresiva, continua y presente en todos los pacientes), rinorrea (acuosa o purulenta si hay sobreinfección micótica o bacteriana) e hipernasalidad que es típica de obstrucción nasal completa asociada con hiposmia o anosmia. Este cuadro suele presentarse al igual que en la rinosinusitis crónica durante un mínimo de doce semanas. ${ }^{2}$ Para su correcta evaluación existen múltiples valoraciones que en forma subjetiva establecen la severidad de los síntomas. De estas la más reconocida y utilizada para estudios clínicos es la escala subjetiva de síntomas de Lund-Mackay, ${ }^{3}$ que los evalúa con puntajes que van de uno a diez de acuerdo con la percepción que tenga el paciente de su síntoma.

La forma correcta de realizar la evaluación clínica de esta entidad es mediante rinoscopia anterior o nasofibrolaringoscopia para determinar la extensión de la enfermedad. El examen endoscópico indica que la mayoría de los pólipos se originan de la mucosa del ostium, fisuras o recesos de los complejos osteomeatales. Para su adecuada descripción se hace necesario un sistema de estadificación subjetivo y referido por el examinador. La herramienta más utilizada para este propósito es la escala endoscópica de Lund-Kennedy, ${ }^{4}$ que de acuerdo con el grado de extensión y la presencia de rinorrea arroja resultados en puntajes de uno a cuatro que corresponden a leve, cinco a ocho cuando hay compromiso moderado y de nueve a doce si es severo.

La TAC de senos paranasales es el estándar de oro en su evaluación objetiva. Se han utilizado múltiples métodos para la descripción y el estadio de los cambios inflamatorios. Para estandarizarlos hay escalas dentro de las cuales la más reconocida y al parecer bien correlacionada con los hallazgos clínicos y endoscópicos es el sistema de estadificación de Kennedy ${ }^{4}$, que de acuerdo con el compromiso unilateral o bilateral y la extensión, arroja resultados desde grado I hasta IV.

El principal objetivo del tratamiento es el alivio sintomático, dado por la obstrucción nasal, congestión, hiposmia y rinorrea. En forma secundaria la terapia intenta disminuir la frecuencia de infecciones, recurrencia de la enfermedad y mejoría de los síntomas respiratorios bajos, así como complicaciones tardías como mucocele y compromiso orbitario. Dentro de las opciones de manejo médico el esteroide tópico nasal y el uso de antihistamínicos son las más frecuentes. Un ciclo corto de esteroide oral está indicado cuando no hay respuesta 
clínica o endoscópica franca. Ante la evidencia de sobreinfección los antibióticos orales están indicados, esto es cefalosporinas de primera generación, betalactámicos y su asociación con inhibidores de betalactamasas. La cirugía endoscópica transnasal es la piedra angular del manejo de la poliposis nasal y se reserva para aquellos casos en que el tratamiento médico no es eficaz y se suele acompañar con corticoterapia pre y posoperatoria para evitar o retrasar en lo posible las recidivas. ${ }^{5}$

Tras la cirugía lo habitual es la recurrencia de la enfermedad. En un estudio reciente ${ }^{6 ; 7}$ luego delaremoción quirúrgica de los pólipos se realizó seguimiento a los pacientes sin otro manejo adicional. Durante los tres años del estudio $53 \%$ de los pacientes requirieron una sola cirugía, $26 \%$ dos, $12 \%$ tres y $9 \%$ cuatro o más El $20 \%$ presentó recurrencia durante el primer mes posoperatorio, tiempo que varió entre un mes y 27 años, con una media de 2.5 años. Datos similares se reportaron en estudios posteriores solocon intervención quirúrgica: $42 \%{ }^{8}$ y $87 \%^{9}$ al año; $53 \%^{10}$ y $59 \%^{11}$ dentro de los primeros cuatro años; $18 \%$ en los primeros cuatro meses; ${ }^{12}$ de $15 \%$ a $17 \%$ de seis meses a 8 años (dependiendo del tipo de cirugía) ${ }^{13}$ y $81 \%$ dentro de los primeros 2 años. ${ }^{14}$ Dada esta alta tasa de recurrencia, la remoción quirúrgica por medio de cirugía endoscópica transnasal no puede considerarse una terapia única o definitiva.

La propuesta principal de este estudio es determinar la frecuencia y el tiempo de recurrencia temprana con una muestrahomogénea de pacientes con poliposis nasal que utilizaron en forma ininterrumpida inhibidores de leucotrieno a dosis estándar de $10 \mathrm{mg} / v i ́ a$ oral/día. Como objetivos secundarios se pretende determinar el grado de control sintomático en quienes utilizan esta medicación en tres aspectos fundamentales que comparten el motivo más frecuente de consulta: rinorrea, prurito y obstrucción nasales.

\section{Métodos}

Estudio de cohorte observacional descriptivo en una población que correspondió a todos los pacientes llevados a cirugía endoscópica transnasal por poliposis nasal en el Hospital de San José de Bogotá DC, entre julio de 2009 y enero de 2011, para ser manejados con montelukast con dosis de $10 \mathrm{mg} /$ día en el posoperatorio. Se denominaron como criterios de inclusión: mayores de 18 años diagnosticados con poliposis nasal (por clínica, TAC y NFL) sin antecedente de cirugía endoscópica transnasal, con cualquier manejo previo para procesos obstructivos nasales, que consintieran seguir el manejo quirúrgico y posterior terapia supervisada con montelukast y asistieran a los controles establecidos por el médico tratante; fueron criterios de exclusión: pacientes con reporte de patología posquirúrgica diferente a poliposis, aquellas en gestación o lactancia, cuando había historia de hipersensibilidad a la aspirina, mastocitosis nasal, fibrosis quística, asma de presentación tardía, inmunodeficiencia o disquinesia primaria, trastomos psiquiátricos documentados por historia clínica, contraindicaciones para la valoración conTAC y los que no aceptaron el consentimientoinformado. No se realizó ningún tipo de muestreo, ya que se trata de todos los que cumplan con los criterios de admisión dentro del período de estudio señalado. Es importante recalcar que el análisis de datos de este informe preliminar se realizó solo con los doce casos que tenían completos los datos de seguimiento hasta el sexto mes posoperatorio.

En cuanto a la información, se tomó del paciente como fuente primaria y en forma secundaria de la historia clínica. El instrumento fue un formato donde se consignaron las variables, como datos personales y de identificación, antecedentes relevantes, un primer cuestionario sobre estado sintomático al momento del ingreso y el espacio para el registro de los hallazgos al examen por NFL inicial y de TAC prequirúrgico. Se registró el tipo de manejo quirúrgico realizado y todos los controles mensuales con la información del uso de la medicación, estado clínico por NFL y sintomático por escala subjetiva de síntomas $^{3}$ (desde 1 hasta 10 , considerando que los síntomas leves corresponden a valores de 1 a 3 , moderados de 4 a 7 y severos de 8 a 10), hasta el sexto mes, momento en el que se realizó control imagenológico con TAC de senos paranasales. En este punto se completó el seguimiento.

El estudio por ser de carácter observacional de una intervención o terapia requirióla supervisión del comité de ética del Hospital de San José, iniciando con la aprobación mediante la aplicación del consentimiento 
informado, el cual fue validado pues contenía información precisa y entendible por el paciente de los posibles beneficios del estudio, sus riesgos, la forma de realización, al igual que el carácter voluntario de su participación y el manejo de los resultados, considerando las cláusulas de privacidad de la información y la no identificación de los pacientes para otro propósito diferente al estudio. Todo esto se ajustó a las recomendaciones delinforme Belmont y las normas intemacionales de la declaración de Helsinki. Se consultó de igual forma la reglamentación lineada por la Resolución 8430 de 1993 en investigación para la República de Colombia considerando el presente estudio como de riesgo mínimo.

El análisis de los datos se realizó en el programa estadístico STATA 10. Se hallaron para todas las variables frecuencias absolutas, porcentajes y luego se practicaron análisis cruzados. Se recurrió al método de Kaplan Meier para establecer función de tiempo desde la cirugía hasta el momento de recurrencia. Se utilizaron gráficos lineales para analizar la evolución del control sintomático a través del tiempo para los tres síntomas evaluados.

\section{Resultados}

La caracterización de la población estudiada hasta el momento correspondiente a doce pacientes arrojó los siguientes resultados: seis (50\%) están dentro del grupo etáreo de 41 a 50 años; tres $(25 \%)$ de 51 a 60 ; dos $(16,6 \%)$ mayores de 60 y solo uno $(8,33 \%)$ menores de 40 años. Edad máxima 69 años, mínima 32 y media de 51 años. El género masculino predominó(58\%). Se evaluó el uso de medicación prequirúrgica (Tabla 1), así como los síntomas preoperatorios y su severidad de acuerdo con las escalas subjetivas (Tabla 2).

Dentro de los criterios para llevar al paciente a cirugía se consideraron tres variables: obstrucción nasal, bloqueo del complejo osteomeatal y presencia de rinosinusitis. El resultado fue positivo parados de las tres variables: $75 \%$ obstrucción nasal y $91 \%$ bloqueo de complejo osteomeatal. Para la variable presencia de rinosinusitis fue negativo pues estuvo presente solo en $41 \%$ de la muestra, aunque esta tendencia pudiera cambiar dada la cercanía a la mitad de los pacientes y que aún no está completa la cohorte.
El resultado en relación con NFL y TAC prequirúrgicos fue el esperado pues la gran mayoría se presentó en estadios avanzados (50\% de los pacientes III y $50 \%$ IV de Kennedy) y para NFL $8 \%$ en nivelleve, $25 \%$ moderado y $66 \%$ severo. En el $83 \%$ se usó esteroide tópico después del segundo control como ocurrió con el montelukast (Tabla 3). Este es un dato relevante en la medida que podría demostrar que el tratamiento continuo con esteroide tópico y antihistamínico está relacionado, como veremos más adelante, con adecuado control sintomático.

En relación con la progresión de los síntomas en términos de severidad según la escala subjetiva, se obtuvieron resultados inferiores a 3 correspondientes a leves en el $75 \%$ desde el primer hasta el sexto control, lo cual indica una evolución satisfactoria (Figuras 1, 2 y 3).

\begin{tabular}{|c|c|}
\hline Característica & $N=12(\%)$ \\
\hline \multicolumn{2}{|c|}{$\begin{array}{l}\text { Uso de medicación } \\
\text { preoperatoria }\end{array}$} \\
\hline \multicolumn{2}{|l|}{ Solución salina } \\
\hline Si & $9(75)$ \\
\hline \multicolumn{2}{|c|}{ Esteroide tópico nasal } \\
\hline Si & $10(83.3)$ \\
\hline \multicolumn{2}{|l|}{ Antihistaminico } \\
\hline Si & $10(83.3)$ \\
\hline \multicolumn{2}{|l|}{ Esteroide tópico } \\
\hline Si & $4(33.3)$ \\
\hline
\end{tabular}

Tabla 2. Frecuencia de síntomas prequirúrgicos y su severidad según escala subjetiva

\begin{tabular}{|c|c|c|c|}
\hline $\begin{array}{l}\text { Escala subjetiva } \\
\text { de síntomas }\end{array}$ & $\begin{array}{l}\text { Rinorrea } \\
n=12 \text { (\%) }\end{array}$ & $\begin{array}{c}\text { Prurito nasal } \\
n=\mid 2(\%)\end{array}$ & $\begin{array}{c}\text { Obstrucción } \\
\text { nasal } \\
n=12(\%)\end{array}$ \\
\hline 1 & $2(16.6)$ & $4(33)$ & 0 \\
\hline 2 & 0 & $2(16.6)$ & $I(8.3)$ \\
\hline 3 & 0 & $\mathrm{I}(8.3)$ & 0 \\
\hline 4 & 0 & $2(16.6)$ & $\mathrm{I}(8.3)$ \\
\hline 5 & $\mathrm{I}(8.33)$ & 0 & $2(16.6)$ \\
\hline 6 & 0 & 0 & 0 \\
\hline 7 & $3(25)$ & $2(16.6)$ & I(8.3) \\
\hline 8 & $2(16.6)$ & I (8.3) & $4(33)$ \\
\hline 9 & 0 & 0 & 0 \\
\hline 10 & $4(33)$ & 0 & $3(25)$ \\
\hline
\end{tabular}




\section{Tabla 3. Esquema de medicación posoperatoria a través del seguimiento}

$\begin{array}{lllllll}\text { Caracteristica } & \text { Control I } & \text { Control } 2 & \text { Control } 3 & \text { Control } 4 & \text { Control } 5 & \text { Control } 6\end{array}$

Uso de esteroide

\begin{tabular}{|c|c|c|c|c|c|c|}
\hline tópico & $\mathrm{N}+12(\%)$ & & & & & \\
\hline Si & $7(58)$ & $10(83)$ & II(91.6) & $12(100)$ & II(9|.6) & $12(100)$ \\
\hline No & $5(41)$ & $2(16)$ & $I(8.3)$ & 0 & I(8.3) & 0 \\
\hline
\end{tabular}

Uso de montelukast

\begin{tabular}{ccccccc} 
Si & $12(100)$ & $12(100)$ & $12(100)$ & $12(100)$ & $12(100)$ & $12(100)$ \\
No & 0 & 0 & 0 & 0 & 0 & 0 \\
\hline
\end{tabular}

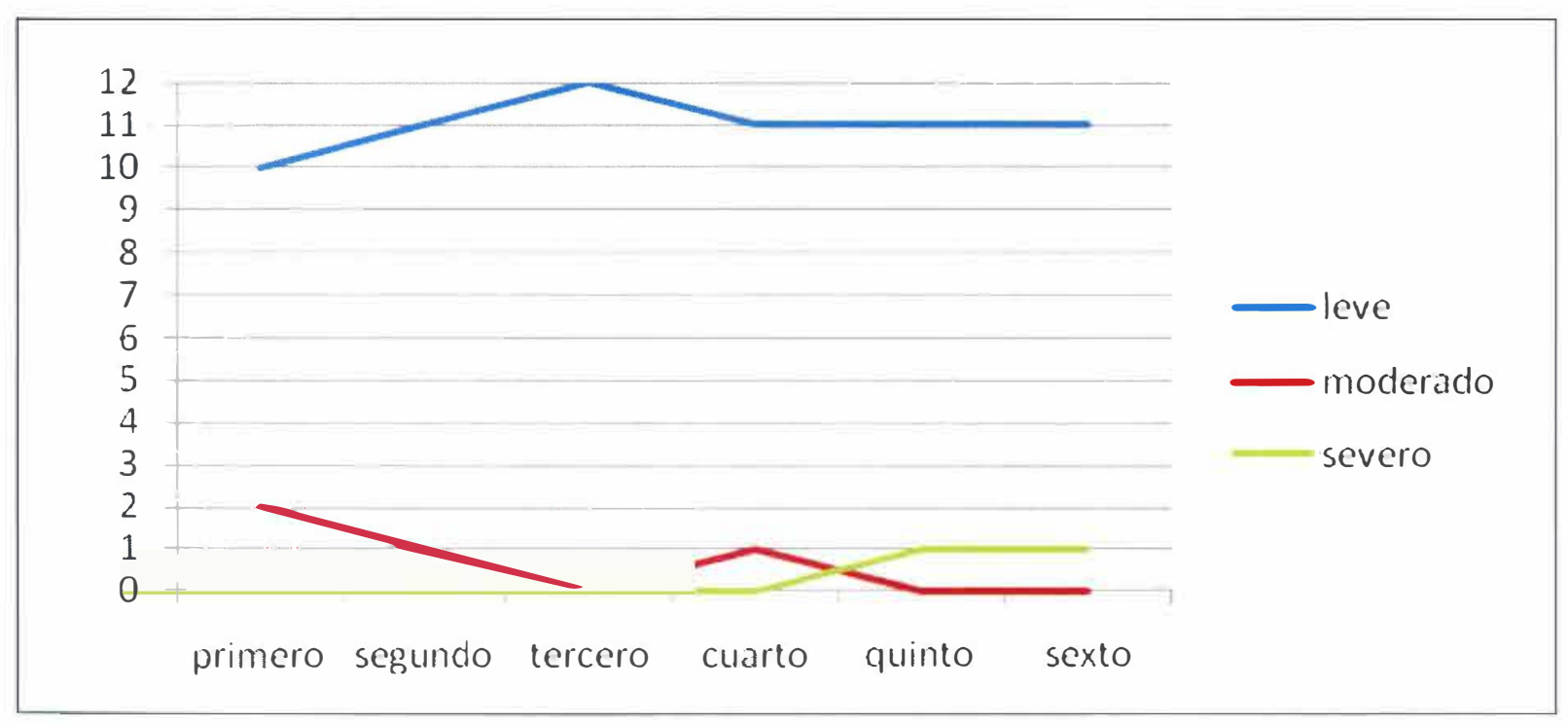

Figura I. Progresión del control sintomático en el seguimiento mensual para prurito nasal según escala subjetiva.

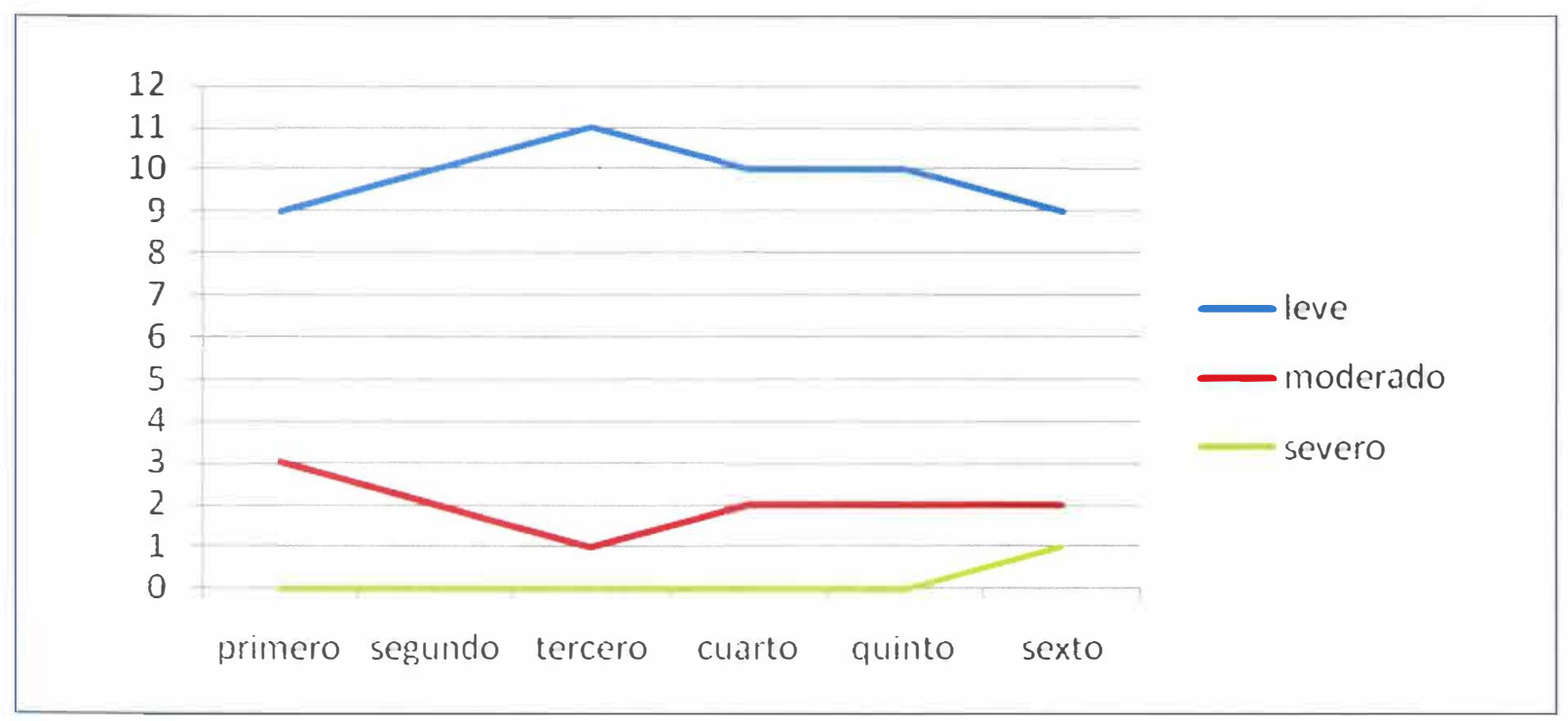

Figura 2. Progresión del control sintomático en el seguimiento mensual para obstrucción nasal según escala subjetiva. 


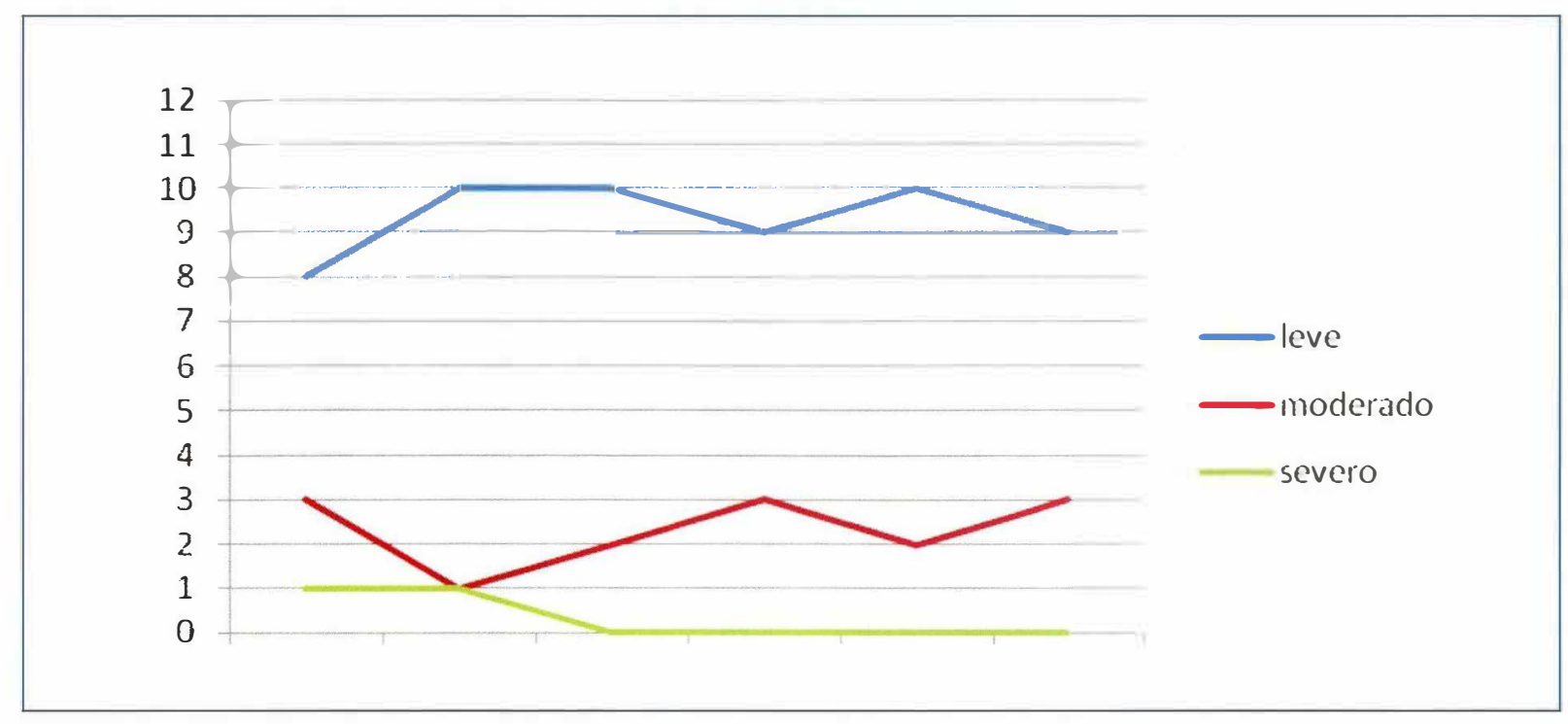

Figura 3. Progresión del control sintomático en el seguimiento mensual para prurito nasal según escala subjetiva.

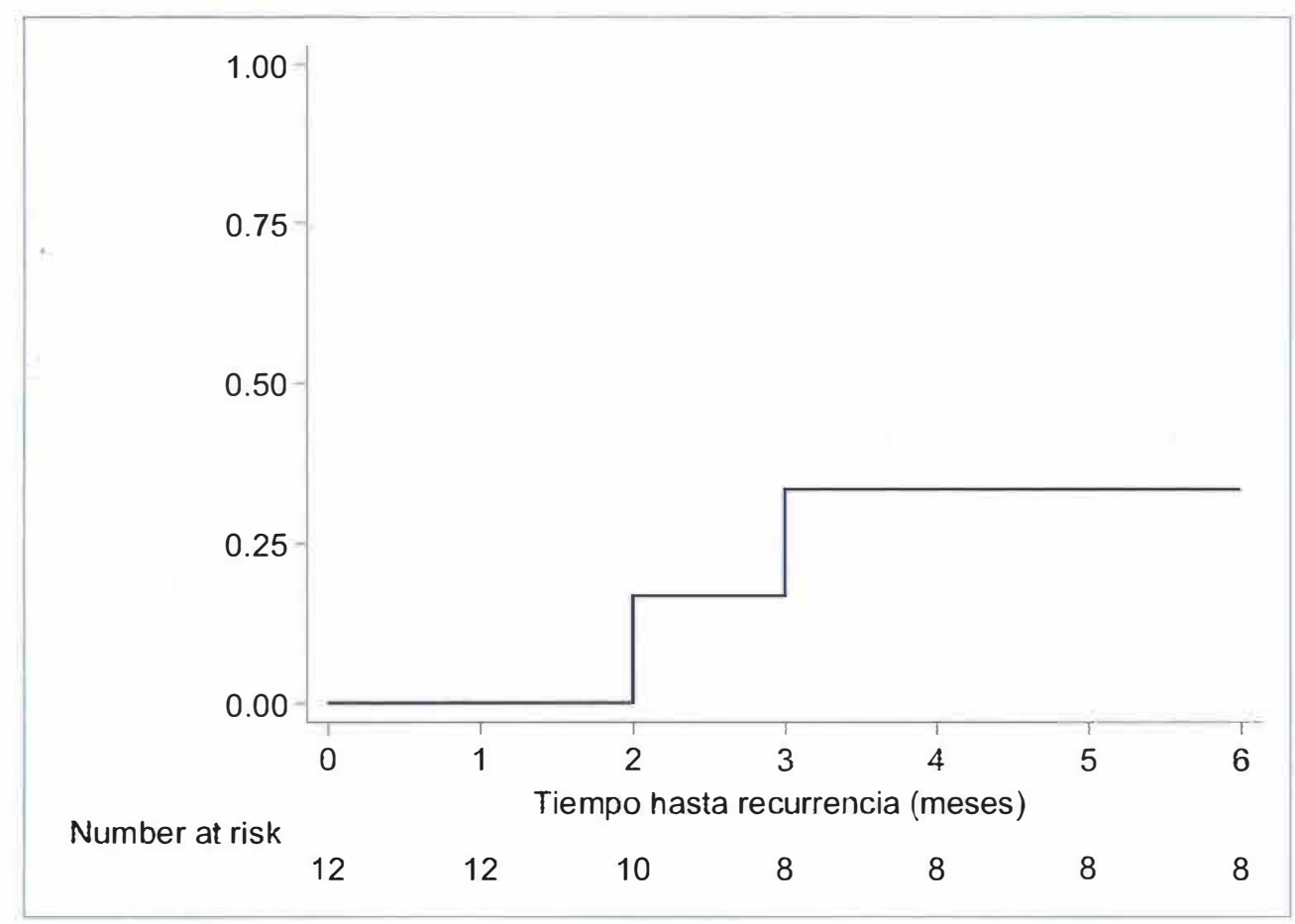

Figura 4. Tiempo de recurrencia y población en riesgo en el posoperatorio.

Por último en relación con la recurrencia y el tiempo de aparición, se determinó temprana a los dos meses de seguimiento en dos pacientes y a los tres meses en un caso), con recurrencia completa en los doce pacientes de $33 \%$ en los seis meses de seguimiento (Figura 4).

\section{Discusión}

Como se anotó antes, la poliposis nasal afecta a cerca del $4 \%$ de la población total en los países occidentales ${ }^{6} \mathrm{y}$ representa un problema para el otorrinolaringólogo debido 
a la severidad, cronicidad y rata de recurrencia. Las características histomorfológicas demuestran que los pólipos corresponden a inflamación eosinofílica y destrucción progresiva del tejido conectivo. $\mathrm{Su}$ tratamiento es un reto para el clínico. Hay una amplia gama de posibilidades en lo relativo a manejo médico. La terapia convencional la lideran diferentes moléculas de esteroide tópico que a dosis regular cada doce horas ha demostrado mejorar la sintomatología en el manejo a largo plazo y al parecer también evita la posible recurrencia de las lesiones, así como ciclos cortos de esteroide oral que utilizados en forma correcta minimizan la posibilidad de efectos adversos. Es importante reconocer que para este manejo sintomático hay otras posibilidadesqueincluyen los antihistamínicos de segunda y tercera generación, los estabilizadores de mastocitos y la furosemida.

En la actualidad los inhibidores de leucotrienos han ganado un papel preponderante en el manejo a largo plazo de procesos inflamatorios crónicos como el asma en vía respiratoria inferior y rinitis alérgica en la superior. ${ }^{7}$ Los conocimientos actuales de la fisiopatología de la poliposis nasal recomiendan esta molécula por su amplio perfil de seguridad como una buena alternativa para el control sintomático de estos pacientes, considerando que muchos de ellos no son atópicos concurrentes. El montelukast (la molécula más difundida y usada en población adulta y niños) ha sido estudiado en múltiples ocasiones y propuesta comomanejo de primera línea en el tratamiento de rinitis alérgica de difícil manejo. Puesto que esta es un elemento clave en la patogenia de la poliposis nasal, nos parece indispensable su uso, no solo en el control sintomático sino también como factor que evite recurrencia, aspecto poco evaluado y considerado. ${ }^{7}$

Ante este panorama y sin evidencia clara de la frecuencia de recurrencia temprana de poliposis o su comportamiento en la población colombiana, después de revisar la literatura en la que se encuentran grandes series americanas y europeas de pacientes operados por poliposis con cirugía endoscópica transnasal, todas con cortos seguimientos o sesgos de confusión que dificultan la evaluación objetiva de los resultados, consideramos útil hacer un seguimiento y caracterización de nuestra población con poliposis, determinar la respuesta al manejo convencional con esteroides tópicos (sin discriminar la molécula utilizada y compararla con aquellas que en forma continua utilicen montelukast.

Aunque el estudio aun está en desarrollo y se continúan recolectando datos para lograr un tamaño adecuado de cohorte, los resultados parciales presentan varios datos interesantes. En primerlugar, en relación con la población afectada la presentación de poliposis nasal es predominante en hombres de edad media (50\% entre 41 y 50 años de edad, 58\% correspondiente al sexo masculino). El uso de medicación prequirúrgica como suero fisiológico nasal, antihistamínicos y esteroides tópicos en el 83\% es común como medicación de elección en procesos alérgicos crónicos y síndromes obstructivos nasales de larga data. Por otro lado, se realizaron ciclos cortos de esteroide oral solo en cuatro de los pacientes (33\%), en razón a que su modo de utilización más frecuente es la preparación prequirúrgica en el IV nivel de atención.

En relación con la estadificación prequirúrgica de la enfermedad, se logró establecer el estado clínico por VFL y radiología al momento del ingreso al estudio, esto es NFL con puntaje superior a siete en la escala de LundKennedy $(66,63 \%)$ corresponde a compromiso moderado y TAC en escala de Kennedy superior a III se ven en estadios bilaterales o difusos.

Tras el manejo quirúrgico, el porcentaje de recurrencia en los doce pacientes con seguimiento de seis meses fue $33 \%$ que se presentó de manera temprana entre el segundo y tercer mes aunque solo fue evidente en TAC posoperatorio a los seis meses en $25 \%$ de los pacientes, lo cual se puede interpretar como no significativa o equivalente a linfedema posoperatorio, lo que a su vez plantea la posibilidad de un sesgo de confusión.

El control de síntomas posoperatorios como obstrucción nasal, rinorrea y prurito fue excelente con escalas subjetivas por debajo de 3 (correspondientes a síntomas leves) en $75 \%$ de los casos. Este control sintomático resulta eficiente en el seguimiento posoperatorio de los pacientes en la medida que refleja su mejoría clínica pero no puede descartarse que corresponda al uso continuado de esteroide tópico nasal que es 
recomendación general en poliposis nasal o inherente al mismo manejo quirúrgico.

Con los resultados obtenidos hasta ahora, la recomendación para manejo de síntomas posoperatorios con montelukast en pacientes con poliposis nasal se mantiene; esto en razón a que en conjunto con el esteroide tópico intranasal el control podría ser completo mejorando la calidad de vida. En relación con la recurrencia, los resultados son promisorios pero su utilidad solo será demostrable o rebatida al completar el seguimiento en la cohorte propuesta inicialmente.

\section{Referencias}

1. Pawliczak R, Lewandowska-Polak A, Kowalski ML. Pathogenesis of nasal polyps: an update. Curr Allergy Asthma Rep. 2005;5:463-71.

2. Nasal polyps: origin, etiology, pathogenesis, and structure. In: Kennedy D, Bolger W. Diseases of the sinuses diagnosis and management. Hamilton : B.C. Decker; 2001.

3. Chiu AG, Kennedy DW. Surgical management of chronic rhinosinusitis and nasal polyposis: a review of the evidence. Curr Allergy Asthma Rep. 2004;4:486-489.
4. Lund V J, Kennedy DW. Staging for rhinosinusitis. Otolaryngol Head Neck Surg. 1997; 117:S35-S40.

5. Llorente JL, Martinez-Farreras A, Rodrigo JP et al. [Nasal polyposis: postoperative long term results (5 years) after endoscopic sinus surgery]. Acta Otorrinolaringol Esp. 2002:53:102-9.

6. Wynn R, Har-El G. Recurrence rates after endoscopic sinus surgery for massive sinus polyposis. Laryngoscope. 2004;114:811-13.

7. Haberal I, Corey JP. The role of leukotrienes in nasal allergy. Otolaryngol Head Neck Surg. 2003;129:274-79.

8. Kirtsreesakul V. Update on nasal polyps: etiopathogenesis. J Med Assoc Thai. 2005;88:1966-72.

9. Klemi PJ, Virolainen E, Puhakka H. Theeffect of in dipropionate on the nasal mucosa. Rhinology. 1980;18:19-24.

10. Blumstein GI, Tuft L. Allergy treatment in recurrent nasal polyposis: its importance and value. Am J Med Sci. 1957;234:269-80.

11. Jantti-Alanko S, Holopainen E, Malmberg H. Recurrence of nasal polyps after surgical treatment. Rhinol Suppl. 1989;8:59-64.:59-64.

12. Wigand ME, Hosemann W. Microsurgical treatment of recurrent nasal polyposis. Rhinol Suppl. 1989;8:25-9.

13. Friedman WH, Katsantonis GP. Intranasal and transanral ethmoidectomy: a 20-yearexperience. Laryngoscope. 1990;100:343-48.

14. Patriarca G, Bellioni P, Nucera E et al. Intranasal treatment with lysine acetylsalicylate in patients with nasal polyposis. Ann Allergy. 1991;67:588-92. 